Animal characteristics. Animals divided in groups \pm succinate analysis performed on animal.

\title{
6-weeks-old rats
}

\begin{tabular}{|c|c|c|c|c|}
\hline & \multicolumn{2}{|c|}{ non-DM } & \multicolumn{2}{|c|}{ ZDF } \\
\hline & $\begin{array}{c}\text { all analysis } \\
(n=12)\end{array}$ & $\begin{array}{c}\text { no succinate } \\
\text { analysis } \\
(n=7)\end{array}$ & $\begin{array}{c}\text { all analysis } \\
(n=16)\end{array}$ & $\begin{array}{c}\text { no succinate } \\
\text { analysis } \\
(n=2)\end{array}$ \\
\hline Bodyweight (g) & $151.2 \pm 24.4$ & $169 \pm 19.8 \mathrm{~ns}$ & $196.2 \pm 16.7$ & $202.5 \pm 3.5 \mathrm{~ns}$ \\
\hline Heartweight (mg) & $651 \pm 115$ & $780 \pm 243 \mathrm{~ns}$ & $725 \pm 177$ & $773 \pm 42 \mathrm{~ns}$ \\
\hline HW/BW ratio & $4.3 \pm 0.4$ & $4.6 \pm 1.2 \mathrm{~ns}$ & $3.7 \pm 0.7$ & $3.8 \pm 0.1 \mathrm{~ns}$ \\
\hline B-glucose (mmol/L) & $4.9 \pm 0.5$ & $4.9 \pm 0.8 \mathrm{~ns}$ & $6.4 \pm 1.4$ & $6.5 \pm 1.1 \mathrm{~ns}$ \\
\hline P-total cholesterol (mmol/L) & $2.2 \pm 0.5$ & $1.8 \pm 0.6 \mathrm{~ns}$ & $2.4 \pm 0.9$ & $1.9 \pm 1.2 \mathrm{~ns}$ \\
\hline P-triglyceride (mmol/L) & $0.5 \pm 0.2$ & $0.4 \pm 0.1 \mathrm{~ns}$ & $1.9 \pm 0.8$ & $2.1 \pm 0.8 \mathrm{~ns}$ \\
\hline
\end{tabular}

12-weeks-old rats

\begin{tabular}{|c|c|c|c|c|}
\hline & \multirow{2}{*}{\multicolumn{2}{|c|}{ non-DM }} & \multirow{2}{*}{\multicolumn{2}{|c|}{ ZDF }} \\
\hline & & & & \\
\hline & $\begin{array}{c}\text { all analysis } \\
\quad(n=14)\end{array}$ & $\begin{array}{c}\text { no succinate } \\
\text { analysis } \\
(n=5)\end{array}$ & $\begin{array}{l}\text { all analysis } \\
\quad(n=16)\end{array}$ & $\begin{array}{c}\text { no succinate } \\
\text { analysis } \\
(n=4)\end{array}$ \\
\hline Bodyweight (g) & $306.2 \pm 13.4$ & $318.8 \pm 11 \mathrm{~ns}$ & $359.8 \pm 21.3 \mathrm{~ns}$ & $365.5 \pm 13.8 \mathrm{~ns}$ \\
\hline Heartweight (mg) & $1061 \pm 109$ & $1106 \pm 127 \mathrm{~ns}$ & $1085 \pm 69 \mathrm{~ns}$ & $1073 \pm 50 \mathrm{~ns}$ \\
\hline HW/BW ratio & $3.5 \pm 0.4$ & $3.5 \pm 0.5 \mathrm{~ns}$ & $3.0 \pm 0.2$ & $2.9 \pm 0.2 \mathrm{~ns}$ \\
\hline B-glucose $(\mathrm{mmol} / \mathrm{L})$ & $5.0 \pm 0.4$ & $4.7 \pm 0.3 \mathrm{~ns}$ & $18.4 \pm 6.4 \mathrm{~ns}$ & $13 \pm 4.3 \mathrm{~ns}$ \\
\hline P-total cholesterol (mmol/L) & $1.8 \pm 0.2$ & $1.4 \pm 0.2 \mathrm{~ns}$ & $3.8 \pm 0.6 \mathrm{~ns}$ & $3.3 \pm 0.8 \mathrm{~ns}$ \\
\hline P-triglyceride (mmol/L) & $0.5 \pm 0.07$ & $0.4 \pm 0.04 \mathrm{~ns}$ & $6.5 \pm 2.0 \mathrm{~ns}$ & $5.3 \pm 2.4 \mathrm{~ns}$ \\
\hline
\end{tabular}

24-weeks-old rats

\begin{tabular}{|c|c|c|c|c|}
\hline & \multirow{2}{*}{\multicolumn{2}{|c|}{ non-DM }} & & \\
\hline & & & \multicolumn{2}{|c|}{ ZDF } \\
\hline & $\begin{array}{c}\text { all analysis } \\
\quad(n=17)\end{array}$ & $\begin{array}{c}\text { no succinate } \\
\text { analysis } \\
(n=2)\end{array}$ & $\begin{array}{l}\text { all analysis } \\
\quad(n=10)\end{array}$ & $\begin{array}{c}\text { no succinate } \\
\text { analysis } \\
(n=8)\end{array}$ \\
\hline Bodyweight (g) & $429.1 \pm 20.8 \mathrm{~ns}$ & $429 \pm 18.4 \mathrm{~ns}$ & $383.4 \pm 30.1 \mathrm{~ns}$ & $423.7 \pm 30.9 \mathrm{~ns}$ \\
\hline Heartweight (mg) & $1431 \pm 341 \mathrm{~ns}$ & $1325 \pm 40 \mathrm{~ns}$ & $1245 \pm 136 \mathrm{~ns}$ & $1414 \pm 537 \mathrm{~ns}$ \\
\hline HW/BW ratio & $3.3 \pm 0.7$ & $3.1 \pm 0.04 \mathrm{~ns}$ & $3.3 \pm 0.3$ & $3.5 \pm 1.5 \mathrm{~ns}$ \\
\hline B-glucose $(\mathrm{mmol} / \mathrm{L})$ & $5.2 \pm 0.3 \mathrm{~ns}$ & $5.8 \pm 0.2 \mathrm{~ns}$ & $17.6 \pm 3.1 \mathrm{~ns}$ & $22.4 \pm 4.2 \mathrm{~ns}$ \\
\hline P-total cholesterol (mmol/L) & $2.5 \pm 1.1 \mathrm{~ns}$ & $2.2 \pm 0.2 \mathrm{~ns}$ & $7.1 \pm 0.9 \mathrm{~ns}$ & $6.4 \pm 1.9 \mathrm{~ns}$ \\
\hline P-triglyceride (mmol/L) & $0.7 \pm 0.2 \mathrm{~ns}$ & $0.6 \pm 0.01 \mathrm{~ns}$ & $7.0 \pm 2.4 \mathrm{~ns}$ & $8.2 \pm 3.9 \mathrm{~ns}$ \\
\hline
\end{tabular}

Mean \pm SD

ns: not significant compared to animal-matched all analysis 